ORIGINAL ARTICLE

\title{
Comparison of clinical outcomes between anteroposterior and lateral penetrating craniocerebral gunshot wounds
}

\author{
Y Izci, H Kayali, M Daneyemez, T Koksel
}

See end of article for

authors' affiliations

Emerg Med J 2005;22:409-410. doi: 10.1136/emj.2004.014704

Correspondence to:

Dr Y Izci, Department of Neurosurgery, Maresal Cakmak Asker Hastanesi, 25100 Yenisehir, Erzurum, Turkey; yusufizci@yahoo. com

Accepted for publication 16 February 2004

\begin{abstract}
Objective: To investigate and compare, using a retrospective clinical study, the clinical outcomes of penetrating craniocerebral gunshot wounds (PCGW) with respect to the trajectory of penetration in the axial plane.

Methods: In total, 22 patients with PCGW caused by conflict, suicide attempt, or accidental firing were included in this study. They were divided into two groups: anteroposterior and lateral. All patients underwent surgical treatment following emergency intervention.

Results: Of the 22 patients, 16 had anteroposterior and 6 had lateral penetrating injury. Four patients with anteroposterior and five patients with lateral injury died despite surgical treatment. Mortality rate was $25 \%$ in the anteroposterior and $83 \%$ in the lateral injury group.

Conclusion: We found that lateral PCGW is the most devastating type of missile injury to the head.
\end{abstract}

C ranial gunshot wounds frequently produce devastating injuries to the central nervous system (CNS) structures. ${ }^{1-3}$ Such wounds are classified as tangential, perforating, and penetrating. Penetrating craniocerebral gunshot wounds (PCGW) are the most devastating type of missile injury to the head. This type of injury, especially if it crosses in the coronal and midline sagittal planes, is usually fatal. ${ }^{4}$

We present 22 cases of PCGW who were treated in our hospital. Depending on the intracranial course of the penetrating agent, we classified the injury types in the axial plane as anteroposterior or lateral. We present a comparison of the clinical outcomes of the two groups and review of the literature.

\section{PATIENTS AND METHODS}

In total, 22 patients with PCGW were treated between 1993 and 2003 at the Department of Neurosurgery, Maresal Cakmak Military Hospital. The patients were evaluated initially by paramedics at the point of injury and transferred to our hospital following administration of first aid.

After the initial neurological examination, the Glasgow Coma Scale (GCS) score of each patient was recorded. All patients underwent radiological examination with plain $x$ ray and computerised tomography (CT) scans. The site of injury, wounding agent, and intracranial lesions were determined and recorded after the radiological evaluation. Infratentorial wounds were excluded from this study. The patients were divided into two groups according to the trajectory of the wounding agent in the cranium; if the agent crossed the brain from left to right or right to left, this injury was classified as lateral (fig 1A), and if the course of the agent was from the anterior part of brain to the posterior or vice versa, it was classified as anteroposterior (fig 1B). Supratentorial anteroposterior and lateral injuries were investigated separately.

All patients underwent surgical treatment including primary closure of the wounds, debridement of necrotic tissues, removal of fragments, and/or duraplasty. Postoperative complications were evaluated and managed in detail. Therapy with broad spectrum antibiotics was given to all patients for 3 days postoperatively. The number of deaths and their causes were recorded for each wound type, and the duration of stay in hospital was determined for each patient. A rehabilitation period was also planned for disabled patients.

\section{RESULTS}

In total, 22 consecutive patients with supratentorial PCGW underwent surgical management over a 10 year period. All the patients were male, mean age 22 years (range 20-30). Of the 22 patients, $15(68 \%)$ were injured during conflict, 5 $(23 \%)$ were suicide attempts and $2(9 \%)$ were injured accidentally. A bullet was the wounding agent in $12(55 \%)$ patients and shrapnel in $10(45 \%)$.

Injury was anteroposterior in 16 patients $(73 \%)$ and lateral in six $(27 \%)$. The wounding agent was a bullet in eight of the $(50 \%)$ of 16 patients with anteroposterior injury and shrapnel in the remaining eight patients. In the group with lateral injury, the wounding agent was a bullet in four cases $(67 \%)$ and shrapnel in the other two.

Mean GCS score on admission was 9 (range 3-15). The GCS score for each group is shown in table 1 . Of 16 patients with anteroposterior injury, 10 (63\%) had a GCS score between 6 and 10, while four $(67 \%)$ of the six patients with lateral injury had a GCS score $<5$.

All patients underwent surgery. Primary closure was performed in $7(32 \%)$ patients, debridement of necrotic tissues and retained fragments in $15(68 \%)$, and debridement in association with duraplasty in $6(27 \%)$.

The result of treatment is summarised in table 1. Nine (41\%) patients died despite all treatment modalities, four $(44 \%)$ of whom of had anteroposterior injury and five (56\%) lateral. All of the patients with GCS score $<5$ on arrival in both groups died. In addition, one patient with anteroposterior injury and GCS score of 7, and one patient with lateral injury and GCS score of 6 died at the end of the first week post-surgery. Diffuse brain damage was the cause of death for seven (78\%) of nine patients. The mortality rate was $25 \%$ among the patients with anteroposterior injury and $83 \%$ for those with lateral injury. Mean duration of stay in the hospital was 17 days (range 1-76 days); 23 days for the patients with anteroposterior injury and 7 days for those with lateral injury.

\section{DISCUSSION}

Of all types of missile wounds to the head, PCGW are the most likely to be fatal. Usually they are caused by high

\footnotetext{
Abbreviations: CNS, central nervous system; $\mathrm{CT}$, computed tomography; GCS, Glasgow Coma Score; PCGW, penetrating craniocerebral gunshot wound
} 

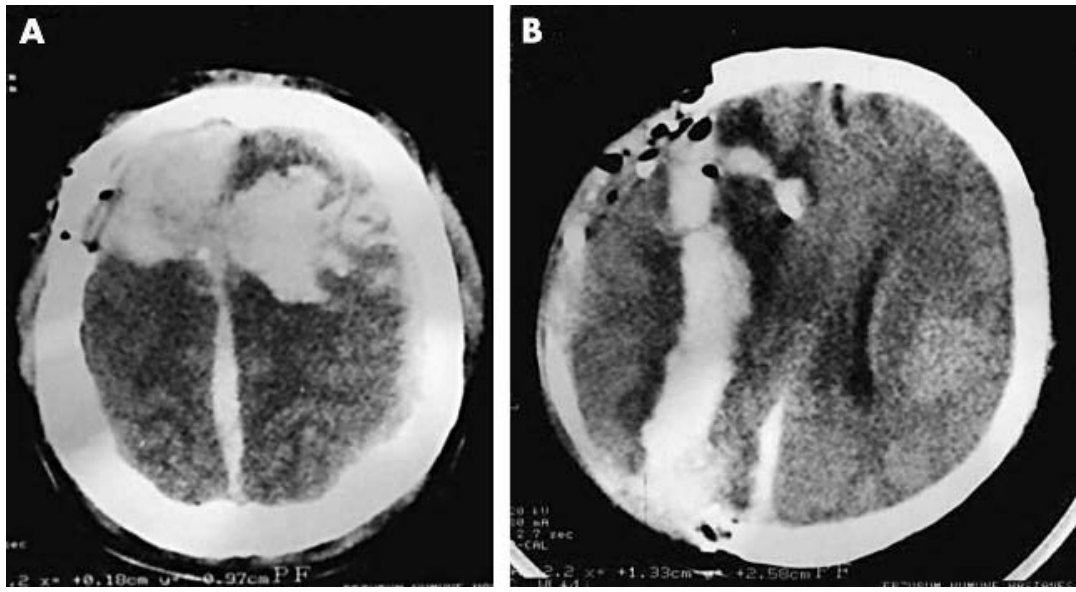

velocity missiles or by handguns fired from a very close range, as in suicide attempts. In the military context, gunshot wounds to the head are characteristically high velocity insults, resulting in a penetrating wound. After the bullet penetrates the outer and inner tables of the skull, it crosses whole brain structures and a percussion wave is transmitted throughout the brain, causing widespread destruction of neuronal cell membranes. ${ }^{4}$ Varying degrees of cavitation in the brain occur along the bullet's path, usually several times larger than the diameter of the bullet. ${ }^{4-7}$ In our series, a bullet was the wounding agent in 50\% of the patients with anteroposterior and $67 \%$ of the patients with lateral injury.

The operative approach was largely the same in both groups - that is, radical debridement. Treatment comprised of four stages; immediate saving of life, prevention of infection, preservation of the nervous tissue, and restoration of anatomical structures.$^{8-10}$ Although there are several different techniques for the surgical management of such injuries, this approach became the de facto standard of treatment for PCGW in our institution.

Consensus is lacking on the prognostic values and limits of salvage of PCGW in the literature. Erdogan et al reported that the presence of diffuse brain damage, brain stem injury, CNS infection, or ventricular injury was associated with poor outcome. ${ }^{4}$ The level of consciousness is also a reliable indicator of severity of injury, correlating predictably with morbidity and mortality. ${ }^{11}{ }^{12}$ The mortality rate increases when the GCS score is $<4 .{ }^{4}$ In our series, the GCS score was $<5$ in the majority of patients with lateral injury, whereas it was 6-10 in most cases of anteroposterior injury. All the patients in both groups with GCS score $<5$ died, which showed that the trajectory of the wounding agent does not affect the prognosis independently of the GCS score on arrival. However, the patients with later injury usually had lower GCS scores at admission.

Ventricular injury is another poor prognostic factor among these patients. The ventricular system is one of the most vulnerable brain sites to damage caused during lateral

Table 1 Distribution of the patients according to GCS scores and type of injury, and results of treatment

\begin{tabular}{|c|c|c|c|c|c|c|}
\hline \multirow[b]{2}{*}{ Type of injury } & \multicolumn{3}{|c|}{$\begin{array}{l}\text { GCS score on } \\
\text { admission }\end{array}$} & \multicolumn{3}{|c|}{ Result of treatment } \\
\hline & $3-5$ & $6-10$ & $11-15$ & Death & Disabled & Well \\
\hline Anteroposterior & 3 & 10 & 3 & 4 & 6 & 6 \\
\hline Lateral & 4 & 1 & 1 & 5 & 1 & 0 \\
\hline
\end{tabular}

GCS, Glasgow Coma Scale.
PCGW. The fragile structure of this system and close proximity to vital structures make it a vital site. ${ }^{413}$ It is difficult to cause a lateral injury without damaging the ventricular system, and therefore high mortality and morbidity rates are inevitable in patients with such injuries. In our series, five of the six patients with lateral injury had ventricular injury with different penetration sites.

The involvement of both cerebral hemispheres was another cause of the poor prognosis in the lateral njury group, whereas only one hemisphere was affected in the anteroposterior injury group, thus the mortality rate was lower. The protection of the other cerebral hemisphere contributed significantly to the prediction of the outcome in patients with anteroposterior injury. This group was also associated with longer hospital stay because of less brain damage compared with the lateral injury group, and required more rehabilitation period.

We observed bihemispheric damage in the patients' brains after lateral PCGW. This rapid destruction of both hemispheres may be relevant to the high mortality rate and shorter hospital stay.

\section{Authors' affiliations}

Y Izci, H Kayali, M Daneyemez, T Koksel, Department of Neurosurgery, Maresal Cakmak Military Hospital, Erzurum, Turkey

Competing interests: none declared

\section{REFERENCES}

1 Brandvold B, Levi L, Feinsod M, et al. Penetrating craniocerebral injuries in the Israeli involvement in the Lebanese conflict 1982-1985. J Neurosurg 1990;72:15-21.

2 Kaufman HH. Civilian gunshot wounds to the head. Neurosurgery 1993;32:962-4.

3 Rish BL, Dillon JD, Weiss GH. Mortality following penetrating craniocerebral injuries. J Neurosurg 1983;59:775-80.

4 Erdogan E, Gonul E, Seber N. Craniocerebral gunshot wounds. Neurosurg Q 2002;12:1-18.

5 Cooper PR, Maravilla K, Cone J. Computerized tomographic scan and gunshot wound to the head: Indications and radiographic findings. Neurosurgery 1979;4:373-80.

6 Pitlyk PJ, Tolchin S, Stewart W. The experimental significance of retained intracranial bone fragments. J Neurosurg 1970;33:19-24.

7 Tsai FY, Huprich JE, Gardner FC, et al. Diagnostic and prognostic implications of computed tomography of head trauma. J Comput Assist Tomogr 1978;2:323-31.

8 Carey M, Young $\mathrm{H}$, Mathis $\mathrm{JL}$, et al. A bacteriological study of craniocerebral missile wounds. J Neurosurg 1971;34:145-54.

9 Dodge PR, Meirowsky AM. Tangential wounds of scalp and skull. J Neurosurg 1952;9:857-60.

10 Hopkinson DAW, Marshall TK. Firearm injuries. Br J Surg 1967;54:344-53.

11 Caveness WF, Meirowsky AM, Rish BL. The nature of posttraumatic epilepsy. J Neurosurg 1979;50:545-53.

12 Meirowsky AM. Secondary removal of retained bone fragments in missile wounds of the brain. J Neurosurg 1982;57:617-21.

13 Gonul E, Baysefer A, Kahraman S, ef al. Causes of infections and management results in penetrating craniocerebral injuries. Neurosurg Rev 1997;20:177-81. 